\title{
Computational Approach to study the effect of point mutations in the development of antifungal resistance to Azoles and Flucytosine Drugs in Candida auris
}

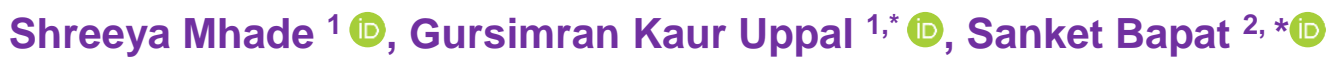

\author{
1 Department of Bioinformatics, Guru Nanak Khalsa College of Arts, Science \& Commerce (Autonomous), Nathalal Parekh \\ Marg, Matunga, Mumbai, Maharashtra-400019, India \\ 2 CSIR-National Chemical Laboratory, Dr Homi Bhabha Rd, Ward No. 8, NCL Colony, Pashan, Pune, Maharashtra-411008, \\ India \\ ${ }^{*}$ Corresponding authors email: gursimran.uppal@gnkhalsa.edu.in; sanket.bapat31@gmail.com \\ DOI: https://doi.org/10.34256/irjmt2144
}

Received: 11-05-2021, Revised: 11-06-2021, Accepted: 12-06-2021, Published: 13-06-2021

Abstract: Background: Candida auris is associated with invasive and severe candidemia, multi-drug resistance and high mortalities. Azoles and Flucytosine are commonly used antifungal drugs. Lanosterol alpha-demethylase (ERG11), Uracil phosphoribosyl transferase (FUR1) are two principal proteins involved in ergosterol biosynthesis and pyrimidine metabolism. However, crystal structures of these proteins from $C$. auris have not yet been established. We constructed structural model of ERG11 and FUR1 proteins for South-African Clade using homology modelling, molecular docking and molecular dynamics simulations. To investigate how point mutations affect drug interaction, we used the same methods on ERG11 mutants (Y132F, K143R) and FUR1 mutants (F211I). Methodology: Homology modelling was used to construct 3D structure of proteins. Reliability of models was analysed by using validation tools. The drug interaction in wild and mutant variants was studied using molecular docking, and binding energy was calculated. Finally, we investigated structural significance of pointmutation between two variants of FUR1 through MD Simulation. Result: Structural models of ERG11 and FUR1 were compared based on binding energy and hydrogen bonding. Few azole compounds showed no effect of mutation on interaction. Further, it was found that binding affinity for 5 -fluorocytosine decreases in the mutant variant of FUR1. MD Simulation of wild variant FUR1-5FC complex showed stabilisation till 7ns while mutated complex was stable for $4.5 \mathrm{~ns}$. Conclusion: $C$. auris resistance to antifungal drugs poses a significant risk to public health. The study sheds light on how drug interactions are influenced by mutations and aids in the development of antifungal drugs.

Keywords: Candida auris, Antifungal Resistance, Point Mutations, Molecular Docking, Molecular Dynamic Simulation

\section{Introduction}

Antimicrobial resistance (AMR) has emerged as one of the leading public health crises of the 21 st century that threatens the effective prevention and treatment of an expanding range of infections [1]. Until the last few decades, AMR has been mainly reported in bacteria. Peculiarly, in medically important Gramnegative ones in which plasmid-mediated or horizontally acquired antibiotic resistance genes are associated with virulence. Prominent genes encoding antibiotic resistance enzymes including extendedspectrum $\beta$-lactamases (ESBLs), carbapenemases, and the MCR colistin resistance gene due to their activity against clinically important antimicrobials agents [2].

However, it is important to realize that AMR is a multi-layered problem and its containment requires a multi-dimensional approach. While resistance in bacteria has been around for decades, a novel multidrug-resistant ascomycetes yeast pathogen belonging to the genus Candida was isolated from the external ear canal of a geriatric female inpatient in a Japanese hospital [3]. It was named as Candida auris as - auris in Latin means 'ears' [3]. [3] who first described this pathogen, found that it clustered in the Metschnikowiaceae clade. The phylogenetic relationship between C.auris and Candida krusei, C. 
lusitaniae, C. haemulonii, C. pseudohaemulonii, and C. duobushaemulonii, which are innately multidrugresistant to amphotericin $B$ (polyenes) and azoles, has been adduced as a reason for the corresponding higher resistance of C. auris to these two drug classes[4-6].

Although Candida auris was initially isolated from external ear canal or discharges of patients with otitis media, subsequent studies show their involvement in fungaemia and other invasive infections with very high associated mortalities and co-morbidities [7]. Contrary to other yeasts, they can be transmitted nosocomially within and between hospitals, patients and the environment. Moreover, Candida auris resistance to at least one antifungal drug such as the azoles (particularly fluconazole and voriconazole), polyenes (amphotericin B), flucytosine, and the echinocandins (caspofungin, micafungin and anidulafungin) is well documented [8-11]. Several studies have established its persistence in clinical environments, including the air and bedding materials, and even in patients undergoing antifungal therapy $[10,12]$. On investigating its virulence and pathogenicity, it was found to be corresponding to or a bit lower than that of Candida albicans [7,13-15].

Presently, C. auris has been reported in around 30 countries on six continents: Single or few cases of Candia auris are recorded from Austria, Belgium, Chile, Costa Rica, Egypt, Greece, Italy, Iran, Norway, Switzerland, Taiwan, Thailand, and the United Arab Emirates. Multiple cases of $\mathrm{C}$. auris have been noted from Australia, Bangladesh, Canada, China, Colombia, France, Germany, India, Israel, Japan, Kenya, Kuwait, Malaysia, the Netherlands, Oman, Pakistan, Panama, Russia, Saudi Arabia, Singapore, South Africa, South Korea, Spain, the United Kingdom, the United States $[16,17]$.

Early diagnosis/detection/identification of Candida auris infections have exhibited to be beneficial as earlier initiation of appropriate antifungal therapy have saved many lives $[18,19]$. However, the inefficacy of several available commercial identification systems/platforms to quickly diagnose C. auris poses as an obstacle to early therapy [8, 20].

Whilst MALDI-TOF MS and RT-PCR are aiding in the diagnosis of $C$. auris with their faster turnaround times, yet, the cost and skills involved in the procuring and operation respectively is a problem for numerous under-resourced mycology laboratories [21-23]. Presently, there are no official therapeutic guidelines, dosage or Clinical Laboratory Standards Institute (CLSI)/European Committee on Antimicrobial Susceptibility Testing (EUCAST) minimum inhibitory concentration (MIC) breakpoints for C. auris infections, and studies evaluating these are scarce [24,25].

Several microscopic, molecular and genomic analysis has documented the presence of phenotypic, phylogenetic and genomic differences between different $C$. auris strains from the same or different regions $[11,26]$. It includes the ability to exist as aggregates or nonaggregate cells, biofilm formation ability, clonality of outbreak strains, phylogenetic and genomic variations between strains from different geographical locations $[13,15]$. There is a great deal to be done to answer a lot of pending questions about this pathogen. Currently, no available antifungal therapy is working against the pathogen as reports of antifungal resistance in Candida auris pop up with each passing day [26]. Yet the effect of mutation as a common contributor to clinical resistance has not been investigated fully. In this context and in view of contributing to mutual interest of combating antimicrobial resistance (AMR), we report the effects of mutations on the interactions of antifungal drugs with their protein targets namely Lanosterol alphademethylase (henceforth termed as ERG11) and Uracil phosphoribosyl transferase (henceforth termed as FUR1) using molecular docking and molecular dynamics simulation studies.

\section{Methodology}

\subsection{Sequence Analysis}

Based on available literature survey, the most common occurring variants of Lanosterol 14-alpha Demethylase and Uracil Phosphoribosyl transferase from the South African Clade of Candida auris was selected for the study. The reviewed 524 and 218 amino acid sequence of Lanosterol 14-alphademethylase (coded by the gene ERG11) and Uracil Phosphoribosyl transferase (coded by the gene FUR1) with NCBI Reference Sequence Acession ID XP_028891800 and XP_028891356 respectively were retrieved from NCBI Protein Database (https://www.ncbi.nlm.nih.gov/protein). These are highly annotated and non-redundant protein sequence.

\subsection{Generation of structural models for proteins}

Three-dimensional (3D) protein structure of native variants of both Lanosterol 14-alphademethylase (ERG11) and Uracil Phosphoribosyltransferase (FUR1) was constructed using homology modelling approach of I-TASSER Server (https://zhanglab.ccmb.med.umich.edu/lTASSER/) [27-29]. The mutated variants of both 
ERG11 and FUR1 were obtained by mutating the residues $\mathrm{Y} 132 \mathrm{~F}, \mathrm{~K} 143 \mathrm{R}$ in ERG11 and F126I in FUR1 respectively using the mutagenesis command 'swapaa' available in UCSF Chimera [30].

\subsection{Protein Structure Validation and Optimisation}

The reliability of the generated protein model (wild and mutated) was verified using Structure Analysis and Verification Server version 5.0 (SAVES) [31-34]. The SAVES 5.0 server integrates analysis from multiple widely-used validation algorithms (such as VERIFY 3D, PROCHECK, ERRAT) taking into account certain geometrical parameters, or topological, to validate goodness-of-fit between model structure and experimental data. Post-validation the protein structures were subjected to energy minimisation performed with the partial implementation of the GROMOS96 force-field in Swiss PDB Viewer (SPDBV) [35]. This optimises the protein structure by repairing distorted geometries by moving atoms to release internal constraints.

\subsection{Docking protocol of the protein-drug complexes}

To investigate the protein-ligand interactions, ligand was docked into the specific site of protein using AutoDock Vina [36]. Receptor Grid were centered based on the active residues mentioned by metaPocket metaserver [37] on analysis the protein structure. Passive residues were automatically defined around active residues. Ligands were flexibly docked in the grid box and the positively docked molecules were ranked based on their docking score. The illustration and visualization of the final docked complex were completed with UCSF Chimera [30]. While the interacting residues in the protein-ligand complex were analysed with LigPlot [38].

\subsection{Molecular Dynamics (MD) Simulation}

Both wild and mutated varianted of docked FUR1 protein with 5-fluorocytosine $(5 F C)$ were subjected to MD Simulation with the CHARMM36 Force-field in GROMACS 5.1 Tool [39] on UNIX System. The simulation was minimised using 5000 steps of Steepest Descent Minimisation Algorithm. The system was later equilibrated at a temperature of $300 \mathrm{~K}$ and a pressure of 1 bar for $2 \mathrm{fs}$. Final production run in Protein-Ligand complex MD Simulation was run for 10 ns in both wild and mutated FUR1 protein complexed with $5 F C$ to study the structural stability and difference in the interaction of both the complexes which were later analysed using RMSD, RMSF calculations for the protein-ligand complex.

\section{Result and Discussion}

\subsection{Homology Modelling}

Since the crystallographic structure of the protein was not readily available in RCSB PDB, tertiary structure prediction methods were employed, namely ab initio-based method using the I-TASSER Server where a single query sequence is used for prediction. Since, the protein secondary structure elements are context dependent. The formation of the a-helices is determined by short-range interactions, whereas the formation of $\beta$-strands is influenced by long-range interactions.

For the given Lanosterol 14-demethylase (ERG11) of Candida auris, the structure was modelled using the template: Chain $A$ of protein templates from RCSB PDB having PDB ID 4k0f, 5jlc and 5eab respectively. The templates used were Lanosterol 14demethylase (ERG11) from Saccharomyces cerevisiae (PDB ID: 5eab; PDB ID: 4k0f however this entry is now obsoleted and superseded to PDB ID: 5eqb) and Candida glabrata (PDB ID: 5jlc).

For the given Uracil phosphoribosyl transferase (FUR1) of Candida auris, the structure was modelled using the template: Chain $A$ of protein templates from RCSB PDB having PDB ID 1bd3, 1i5e and 1jlr respectively and Chain $D$ of template with PDB ID 1bd3. The templates used were Uracil phosphoribosyl transferase (FUR1) from Toxoplasma gondii (Chain A of PDB ID: 1jlr; Chain A and D of PDB ID: $1 \mathrm{bd} 4$ ) and Bacillus caldolyticus (PDB ID: 1i5e).

\subsection{Protein Structure Evaluation}

The homology model generated for ERG11 and FUR1 needs to be evaluated to make sure that the structural features of the model are consistent with the physiochemical rules. This evaluation involves checking anomalies in $\Phi-\Psi$ angles with Ramachandran Plot, bond lengths, close contacts, and so on. Further, by comparing the statistical parameters with the constructed model, it reveals which regions of a sequence appear to be folded normally and which regions do not. On evaluation of the modelled structure of ERG11 and FUR1 in Candida auris, it was found that $83.59 \%$ and $90.83 \%$ of the residues in ERG11 and FUR1 respectively had an averaged 3D-1D score more than or equal to 0.2 (computed using VERIFY3D), overall quality of the models has a quality factor of 92.8295 and 91.7476 respectively (computed using ERRAT). To summarise, the generated models were 
correct, validated and could be used for further docking analysis.

\subsection{Molecular Docking Analysis}

To explore how the binding potential differs for various Azoles molecules and Flucytosine molecule for ERG11 and FUR1 protein respectively, we performed a molecular docking studies as summed up in Table 1 and 2. The findings of a current study consist of comparison of molecular docking of azole and flucytosine drug with both wild and mutated variants of ERG11 and FUR1 respectively. Mutation of $\mathrm{Y} 132 \mathrm{~F}$ and $\mathrm{K} 143 \mathrm{R}$ affect the binding of azole to the ERG11 protein as the $\mathrm{Y} 132 \mathrm{~F}$ is one of the active interacting residues while $\mathrm{K} 143 \mathrm{R}$ is a passive residue around the binding pocket. Contrastingly, the same pattern is not observed in all of the azoles i.e. among the 19 Azole compounds, Itraconazole, Posconazole and Clotrimazole are some of the examples which exhibit highest binding potential and no change in the binding affinity even on mutagenesis. Additional studies are needed to probe into this aspect.

We did not delve more into the effect of mutations in ERG11 as the same has been extensively studied since the discovery of antifungal resistance in Candida auris.

Table 1. Molecular docking analysis of Lanosterol alpha-demethylase (ERG11) with selected azole compounds. The binding affinities were calculated using AutoDock Vina docking tool.

\begin{tabular}{|c|c|c|c|}
\hline Sr No. & Compounds & $\begin{array}{l}\text { Lanosterol alpha demethylase } \\
\text { (ERG11) (kcal/mol) }\end{array}$ & $\begin{array}{l}\text { Mutated Lanosterol alpha demethylase } \\
\qquad(\mathrm{mERG} 11)(\mathrm{kcal} / \mathrm{mol})\end{array}$ \\
\hline 1 & Fluconazole & -7.7 & -7.6 \\
\hline 2 & Voriconazole & -9.2 & -8.4 \\
\hline 3 & Itraconazole & -11.2 & -11.3 \\
\hline 4 & Posconazole & -10.9 & -11.3 \\
\hline 5 & Oxiconazole & -8.5 & -8.6 \\
\hline 6 & Terconazole & -8.1 & -10.2 \\
\hline 7 & Clotrimazole & -10.5 & -10.5 \\
\hline 8 & Butoconazole & -7.6 & -8.2 \\
\hline 9 & Tioconazole & -7.7 & -7.7 \\
\hline 10 & Ketoconazole & -8.0 & -10.5 \\
\hline 11 & Miconazole & -8.1 & -8.4 \\
\hline 12 & Econazole & -8.4 & -8.0 \\
\hline 13 & Sertaconazole & -8.7 & -8.8 \\
\hline 14 & Bifonazole & -9.2 & -9.3 \\
\hline 15 & Sulconazole & -8.3 & -8.4 \\
\hline 16 & Isoconazole & -6.8 & -8.4 \\
\hline 17 & Efinaconazole & -8.9 & -8.9 \\
\hline 18 & Isavuconazole & -9.4 & -8.3 \\
\hline 19 & Luliconazole & -7.7 & -7.6 \\
\hline
\end{tabular}

Table 2. Molecular docking analysis of Uracil phosphoribosyl transferase (FUR1) with 5-fluorocytosine. The binding affinities were calculated using Auto Dock Vina docking tool.

\begin{tabular}{|c|c|c|c|}
\hline Sr No. & Compounds & $\begin{array}{c}\text { Uracil phosphoribosyl transferase } \\
\text { (FUR1) }(\mathrm{kcal} / \mathrm{mol})\end{array}$ & $\begin{array}{c}\text { Mutated Uracil phosphoribosyl transferase } \\
(\mathrm{mFUR} 1)(\mathrm{kcal} / \mathrm{mol})\end{array}$ \\
\hline 1 & 5 -fluorocytosine & -4.4 & -4.0 \\
\hline
\end{tabular}




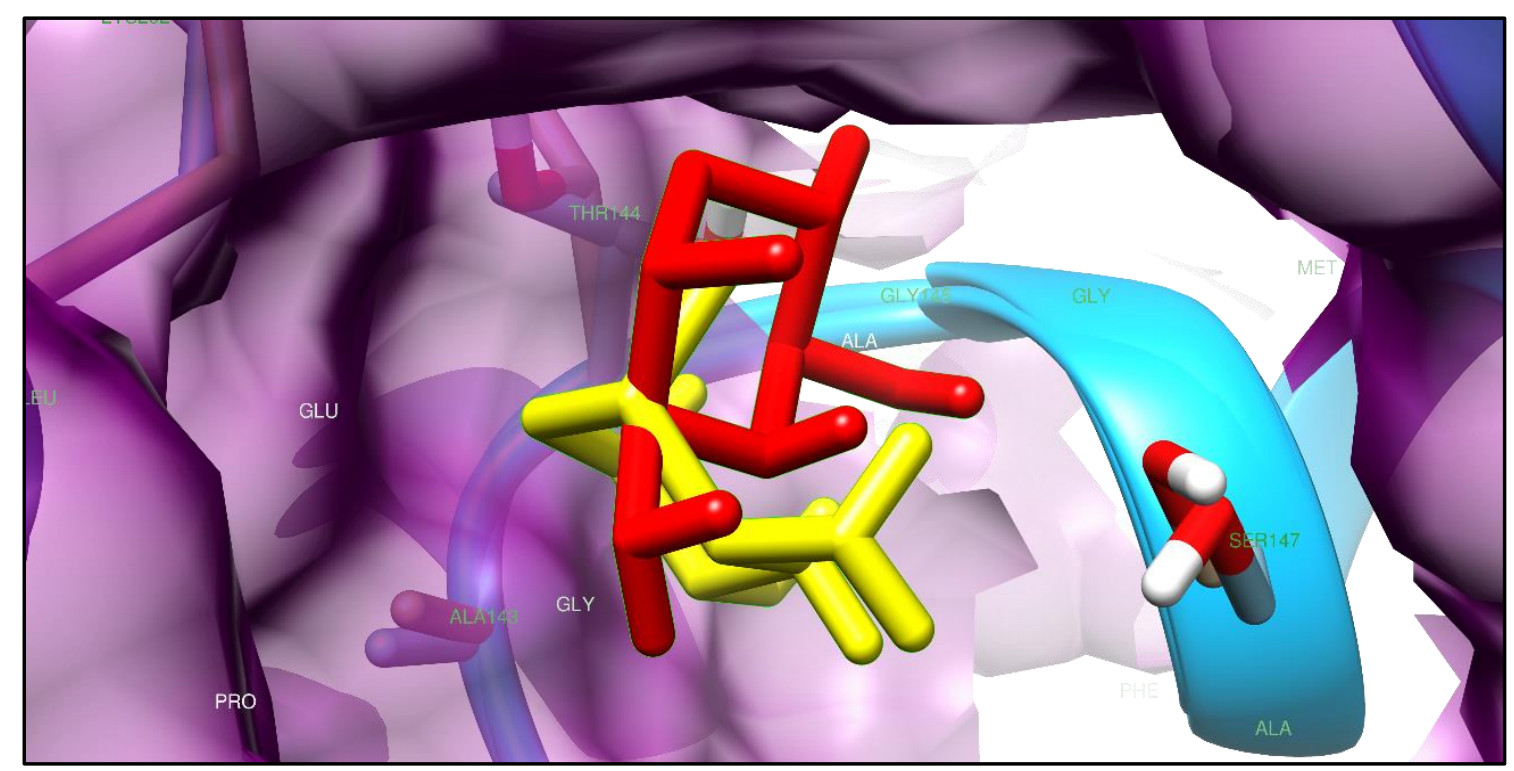

Figure 1. Surface View and conformation of Compound 5-fluorocytosine (5FC) in red colour (wild) and yellow colour (mutated) in the binding pocket of the receptor FUR1 protein.

We postulated that the resistance is due to the overuse of antifungal drugs given in Candida infections, without understanding the aetiology of the species.

Since effect of mutations in FUR1 is still an unexplored arena, our later part of the study focusses on understanding the same. Rhodes et al. (2018)[40] identified a point mutation F211l in FUR1 in a flucytosine resistant Candida auris strain. In our study, we observed a decrease in the binding potential when the ligand was docked with the mutated protein. Hence, FUR1 protein complexed with 5FC was selected for further investigation to ascertain the role of mutation in affecting the binding potential and thereby impacting antifungal resistance to Flucytosine Drugs. The analysis resulted reflected the reaction differences between protein and ligands in the two docking methods. It showed that although the types and number of hydrogen bonds in the two complexes were nearly the same, a plunge in the binding affinity of $5 \mathrm{FC}$ to mutated protein was observed.

We also observed a change in the conformation of 5 -fluorocytosine (5FC) in the binding pocket of the wild and mutated receptor FUR1 protein as shown in Figure 1. In the wild FUR1 protein, the compound 5FC is bound to the Thr144 and Ser147 residues present in the pocket region of the receptor at a distance of $2.75 \AA$ for Thr1 144 and $2.20 \AA$ and $3.03 \AA$ for Ser147 residue. In the mutated FUR1 protein, the compound 5FC is bound to Met141, Ala143 and Gly145 residues at a distance of $2.87 \AA, 2.51 \AA$, and $3.06 \AA$ each apart from Thr144 residue as observed in wild protein. However, interaction with Ser147 is not observed in the mutated protein and it may be due to the effect of point mutation (F126I) in the protein receptor.

\subsection{Molecular Dynamic Simulation}

Molecular Dynamic Simulation Studies were performed to further investigate the differences in the dynamicity and fluctuations of the ligand at the atomic level in the active sites of the wild and mutated FUR1 protein. Figure 2 shows the RMSD trajectories for both wild and mutated variants of FUR1 protein. In case of the wild protein, it reached equilibration and stabilised till $7 \mathrm{~ns}$ in the binding pocket and then the ligand dissociates from the protein receptor while the mutated protein stabilised only till $4.5 \mathrm{~ns}$ in the binding pocket and later binds to other region of the receptor before completely dissociating from the protein.

An early plunge and differences in the stabilisation time observed in the RMSD analysis after MD Simulation of the both complexes explain the effect of point mutation (F211I) in the drug interaction. In the mutated protein complex, an early peak and stabilisation in the binding pocket continues only till 4.5 ns after which a drop is observed and the complex restabilises again although away from the binding pocket till $8 \mathrm{~ns}$ and then ligand completely dissociates from the receptor by the end of the simulation. This early dissociation ascertains that mutation affects the structural stability of the protein which leds to the early release of ligand from the binding pocket. Also, binding of the ligand away from the actual active site of the proteins explains why the clinical specimens of Candida auris are unresponsive towards Flucytosine drugs. 


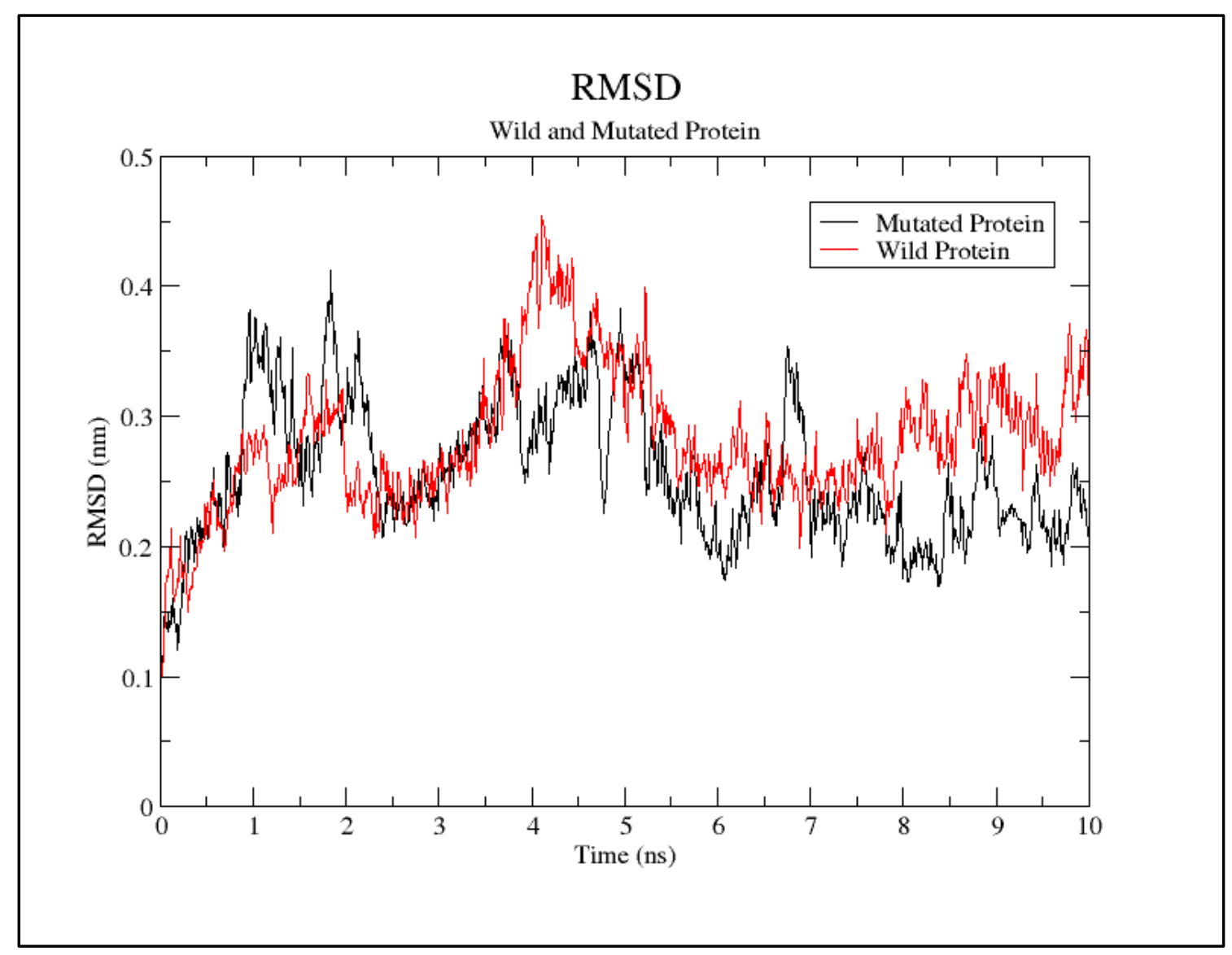

Figure 2. RMSD Analysis: Root Mean Square Deviation of Compound Trajectory for Wild and Mutated variant of FUR1 protein. The RMSD value indicates stability of compound molecules in the pocket region.

\section{Conclusion}

Previous research has shown that mutations in the South African clade's ERG11 Y132F and K143R and F211I in FUR1 increase drug resistance based on clinical data. Because of the similarities between Candida auris and Candida albicans, Candida auris infections are always and yet treated with common antifungal drugs. The failure to recognise the importance of studying infections all the way down to their aetiology has undoubtedly contributed to antifungal resistance. The lack of novel antifungal drugs in development, combined with the emergence of antifungal resistance, necessitates the development of new strategies. Using open-access bioinformatics tools, we attempted to provide important insights into the effect of point mutations in the hotspot region and the underlying mechanism of drug resistance.

No massive difference in binding affinity or structural changes in the binding pocket are observed in both variants of ERG11. Rising number of azole resistance incidences is a result of overuse of common azole drugs. In the FUR1 protein, mutations had a significant impact on binding affinity as well as the structural conformation of the protein's binding pocket. Molecular Dynamic Simulation confirms the early destabilisation of the protein-ligand complex due to a change in pocket conformation. The organism becomes resistant to Flucytosine because there is no significant time for the drug to exert an effect on the protein due to a lack of correct binding and residual interaction.

Finally, using bioinformatics tools to investigate emerging fungal pathogens brings with it new challenges and considerations. These pathogens are frequently understudied, with only a limited amount of annotated genomic, proteomic, and structural data available. A variety of structural bioinformatics techniques, such as homology modelling, protein-ligand docking, and molecular dynamic simulations, in our study. While our study focuses on ERG11 and FUR1 from Candida auris, the findings we present, as well as the considerations and challenges we discuss, can help to pave the way for the development of effective novel antifungal therapeutics that can be used against Candida auris. 


\section{References}

[1] F. Prestinaci, P. Pezzotti, A. Pantosti, Antimicrobial resistance: A global multifaceted phenomenon, Pathogens and global health, 109 (2015) 309-318. [DOI] [PubMed]

[2] P. Nordmann, L. Poirel, The difficult-to-control spread of carbapenemase producers among Enterobacteriaceae worldwide, Clinical Microbiology and Infection, 20 (2014) 821-830. [DOl] [PudMed]

[3] K. Satoh, K. Makimura, Y. Hasumi, Y. Nishiyama, K. Uchida, H. Yamaguchi, Candida auris sp. nov., a novel ascomycetous yeast isolated from the external ear canal of an inpatient in a Japanese hospital, Microbiology and immunology, 53 (2009) 41-44. [DOI] [PubMed]

[4] E. Cendejas-Bueno, A. Kolecka, A. AlastrueyIzquierdo, B. Theelen, M. Groenewald, M. Kostrzewa, M. Cuenca-Estrella, A. GómezLópez, T. Boekhout, Reclassification of the Candida haemulonii complex as Candida haemulonii (C. haemulonii group I), C. duobushaemulonii sp. nov. (C. haemulonii group II), and C. haemulonii var. vulnera var. nov.: Three multiresistant human pathogenic yeasts, Journal of clinical microbiology, 50 (2012) 3641-3651. [DOI] [PubMed]

[5] A.J. Lepak, M. Zhao, E.L. Berkow, S.R. Lockhart, D.R. Andes, Pharmacodynamic optimization for treatment of invasive Candida auris infection, Antimicrob Agents Chemother, 61 (2017) e00791-17. [DOl] [PubMed]

[6] E.L. Berkow, S.R. Lockhart, Fluconazole resistance in Candida species: A current perspective, Infection and drug resistance, 11 (2017) 237-245. [DOl] [PubMed]

[7] R. Ben-Ami, J. Berman, A. Novikov, E. Bash, Y. Shachor-Meyouhas, S. Zakin, Y. Maor, J. Tarabia, V. Schechner, A. Adler, T. Finn, Multidrug-Resistant Candida haemulonii and C. auris, Tel Aviv, Israel, Emerging infectious diseases, 23 (2017) 195-203. [DOl] [PubMed]

[8] European Centre for Disease Prevention and Control, (2016) Rapid Risk Assessment. Candida auris in healthcare settings-Europe.

[9] S.M. Rudramurthy, A. Chakrabarti, R.A. Paul, P. Sood, H. Kaur, M.R. Capoor, A.J. Kindo, R.S.K. Marak, A. Arora, R. Sardana, S. Das, D. Chhina, A. Patel, I. Xess, B. Tarai, P. Singh, A. Ghosh, Candida auris candidaemia in Indian
ICUs: Analysis of risk factors, Journal of Antimicrobial Chemotherapy, 72 (2017) 17941801. [DOl] [PubMed]

[10] S. Schelenz, F. Hagen, J.L. Rhodes, A. Abdolrasouli, A. Chowdhary, A. Hall, L. Ryan, J. Shackleton, R. Trimlett, J.F. Meis, D. Armstrong-James, M.C. Fisher, First hospital outbreak of the globally emerging Candida auris in a European hospital, Antimicrobial Resistance \& Infection Control, 5 (2016) 1-7. [DOI] [PubMed]

[11] S. Tsay, R.M. Welsh, E.H. Adams, N.A. Chow, L. Gade, E.L. Berkow, E. Poirot, E. Lutterloh, M. Quinn, S. Chaturvedi, J. Kerins, S.R. Black, S.K. Kemble, P.M. Barrett, K. Barton, D.J. Shannon, K. Bradley, S.R. Lockhart, A.P. Litvintseva, H. Moulton-Meissner, A. Shugart, A. Kallen, S. Vallabhaneni, T.M. Chiller, B.R. Jackson, Notes from the Field: Ongoing Transmission of Candida auris in Health Care Facilities - United States, June 2016-May 2017, MMWR. Morbidity and mortality weekly report, 66 (2017) 514. [DOI] [PubMed]

[12] S. Vallabhaneni, A. Kallen, S. Tsay, N. Chow, R. Welsh, J. Kerins, S.K. Kemble, M. Pacilli, S.R. Black, E. Landon, J. Ridgway, T.N. Palmore, A. Zelzany, E.H. Adams, M. Quinn, S. Chaturvedi, J. Greenko, R. Fernandez, K. Southwick, E.Y. Furuya, D.P. Calfee, C. Hamula, G. Patel, P. Barrett, P. Lafaro, E.L. Berkow, H. M. Meissner, J. N. Wang, R. P. Fagan, B. R. Jackson, S.R. Lockhart, A. P. Litvintseva, T. M. Chiller, Investigation of the first seven reported cases of Candida auris, a globally emerging invasive, multidrug-resistant fungus-United States, May 2013-August 2016, Morbidity and Mortality Weekly Report, 65 (2016) 1234-1237.

[13] A.M. Borman, A. Szekely, E.M. Johnson, Comparative Pathogenicity of United Kingdom Isolates of the Emerging Pathogen Candida auris and Other Key Pathogenic Candida Species, MSphere, 1 (2016). [DOl] [PubMed]

[14] E. Larkin, C. Hager, J. Chandra, P.K. Mukherjee, M. Retuerto, I. Salem, L. Long, N. Isham, L. Kovanda, K. Borroto-Esoda, S. Wring, D. Angulo, M. Ghannoum, The emerging pathogen Candida auris: Growth phenotype, virulence factors, activity of antifungals, and effect of SCY-078, a novel glucan synthesis inhibitor, on growth morphology and biofilm formation, Antimicrob. 
Agents Chemother, 61 (2017) e02396-16. [DOI] [PubMed]

[15] L. Sherry, G. Ramage, R. Kean, A. Borman, E.M. Johnson, M.D. Richardson, R. RautemaaRichardson, Biofilm-forming capability of highly virulent, multidrug-resistant Candida auris, Emerging infectious diseases, 23 (2017) 328331. [DOI] [PubMed]

[16] Centers for Disease Control and Prevention, (2019) Tracking Candida auris: Candida auris Fungal Diseases CDC, Centers Dis. Control Prev.

[17] A. Chowdhary, C. Sharma, J.F. Meis, Candida auris: A rapidly emerging cause of hospitalacquired multidrug-resistant fungal infections globally, PLOS pathogens, $13 \quad$ (2017) e1006290. [DOI] [PubMed]

[18] A. Chowdhary, V.A. Kumar, C. Sharma, A. Prakash, K. Agarwal, R. Babu, K.R. Dinesh, S. Karim, S.K. Singh, F. Hagen, J. F. Meis, Multidrug-resistant endemic clonal strain of Candida auris in India, European journal of clinical microbiology \& infectious diseases, 33 (2014) 919-926. [DOI] [PubMed]

[19] B. Todd, Clinical alert: Candida auris, American Journal of Nursing, 117 (2017) 53-55. [DOI] [PubMed]

[20] M. Kordalewska, Y. Zhao, S.R. Lockhart, A. Chowdhary, I. Berrio, D.S. Perlina, Rapid and accurate molecular identification of the emerging multidrug-resistant pathogen Candida auris, Journal of clinical microbiology, 55 (2017) 2445-2452. [DOI] [PubMed]

[21] S. Kathuria, P.K. Singh, C. Sharma, A. Prakash, A. Masih, A. Kumar, J.F. Meis, A. Chowdhary, Multidrug-resistant Candida auris misidentified as Candida haemulonii: Characterization by matrix-assisted laser desorption ionization-time of flight mass spectrometry and DNA sequencing and its antifungal susceptibility profile variability by vitek 2, CLSI broth microdilution, and Etest method, Journal of clinical microbiology, 53 (2015) 1823-1830. [DOI] [PubMed]

[22] A. Prakash, C. Sharma, A. Singh, P. Kumar Singh, A. Kumar, F. Hagen, N.P. Govender, A.L. Colombo, J.F. Meis, A. Chowdhary, Evidence of genotypic diversity among Candida auris isolates by multilocus sequence typing, matrix-assisted laser desorption ionization time-of-flight mass spectrometry and amplified fragment length polymorphism, Clinical
Microbiology and Infection, 22 (2016) 1-9. [DOI] [PubMed]

[23] M.C. Arendrup, A. Prakash, J. Meletiadis, C. Sharma, A. Chowdhary, Comparison of EUCAST and CLSI Reference Microdilution MICs of Eight Antifungal Compounds for Candida auris and Associated Tentative Epidemiological Cutoff Values, Antimicrobial agents and chemotherapy, 61 (2017) e0048517. [DOI] [PubMed]

[24] A.J. Lepak, M. Zhao, E. Berkow, S. Lockhart, D.R. Andes, Pharmacodynamic Optimization for the Treatment of Invasive Candida auris Infection, In Open Forum Infectious Diseases, 4 (2017) 73. [DOI]

[25] S.R. Lockhart, K.A. Etienne, S. Vallabhaneni, J. Farooqi, A. Chowdhary, N.P. Govender, A.L. Colombo, B. Calvo, C.A. Cuomo, C. A. Desjardins, E.L. Berkow, M. Castanheira, R.E. Magobo, K. Jabeen, R.J. Asghar, J.F. Meis, B. Jackson, T. Chiller, A.P. Litvintseva, Simultaneous emergence of multidrug resistant Candida auris on three continents confirmed by whole genome sequencing and epidemiological analyses, Clinical Infectious Diseases, 64 (2017) 134-140. [DOI] [PubMed]

[26] J. Yang, R. Yan, A. Roy, D. Xu, J. Poisson, Y. Zhang, The I-TASSER suite: Protein structure and function prediction, Nature methods, 12 (2014) 7-8. [DOl] [PubMed]

[27] Y. Zhang, I-TASSER server for protein 3D structure prediction, BMC Bioinformatics. 9 (2008) 1-8. [DOI] [PubMed]

[28] A. Roy, A. Kucukural, Y. Zhang, I-TASSER: A unified platform for automated protein structure and function prediction, Nature protocols, 5 (2010) 725-738. [DOl] [PubMed]

[29] E.F. Pettersen, T.D. Goddard, C.C. Huang, G.S. Couch, D.M. Greenblatt, E.C. Meng, T.E. Ferrin, UCSF Chimera - A visualization system for exploratory research and analysis, Journal of computational chemistry, 25 (2004) 16051612. [DOI] [PubMed]

[30] J.U. Bowie, R. Lüthy, D. Eisenberg, A method to identify protein sequences that fold into a known three-dimensional structure, Science. 253 (1991) 164-70. [DOI] [PubMed]

[31] R. Lüthy, J.U. Bowie, D. Eisenberg, Assessment of protein models with threedimensional profiles, Nature, 356 (1992) 83-85. [DOI] [PubMed] 
[32]

C. Colovos, T.O. Yeates, Verification of protein structures: Patterns of nonbonded atomic interactions, Protein science, 2 (1993) 15111519. [DOI] [PubMed]

[33] R.A. Laskowski, M.W. MacArthur, D.S. Moss, J.M. Thornton, PROCHECK: a program to check the stereochemical quality of protein structures, Journal of applied crystallography, 26 (1993) 283-291. [DOl] [PubMed]

[34] N. Guex, M.C. Peitsch, SWISS-MODEL and the Swiss-PdbViewer: An environment for comparative protein modeling, Electrophoresis, 18 (1997) 2714-2723. [DOl] [PubMed]

[35] T. Oleg, O.J. Arthur, AutoDock Vina: Improving the Speed and Accuracy of Docking with a New Scoring Function, Efficient Optimization, and Multithreading, Journal of computational chemistry, 31 (2010) 455-461. [DOI] [PubMed]

[36] B. Huang, Metapocket: A meta-approach to improve protein ligand binding site prediction, OMICS A Journal of Integrative Biology, 13 (2009) 325-330. [DOI] [PubMed]

[37] R.A. Laskowski, M.B. Swindells, LigPlot+: Multiple ligand-protein interaction diagrams for drug discovery, Journal of Chemical Information and Modeling, 51 (2011) 27782786. [DOI] [PubMed]

[38] D. Van Der Spoel, E. Lindahl, B. Hess, G. Groenhof, A.E. Mark, H.J.C. Berendsen, GROMACS: Fast, flexible, and free, Journal of computational chemistry, 26 (2005) 1701-1718. [DOI] [PubMed]

[39] J. Rhodes, A. Abdolrasouli, R.A. Farrer, C.A. Cuomo, D.M. Aanensen, D. Armstrong-James, M.C. Fisher, S. Schelenz, Genomic epidemiology of the UK outbreak of the emerging human fungal pathogen Candida auris article, Emerging microbes \& infections, 7 (2018) 1-12. [DOI] [PubMed]

\section{Acknowledgement}

The authors are grateful to the Department of Bioinformatics and the Principal of G N Khalsa College for the infrastructure and support.

\section{Funding}

No funding was received for conducting this study.

\section{Conflict of interest}

None of the authors have any conflicts of interet to declare.

\section{Data Deposition:}

Mhade, Shreeya; Kaur Uppal, Gursimran; Bapat, Sanket (2020), "Computational Approach to study the effect of point mutations in the development of antifungal resistance to Azoles and Flucytosine Drugs in Candida auris", Mendeley Data, v1

http://dx.doi.org/10.17632/gd9vtc3k7j.1\#file-

262b2af8-f1ea-4461-a7bc-6ce640b053c7

Author Contribution:

Shreeya Mhade: Data curation, Investigation, Visualization, Writing - Original draft preparation; $\mathrm{Dr}$ Gursimran Kaur Uppal: Project administration; Supervision; Writing - review \& editing; Dr Sanket Bapat: Conceptualization; Methodology; Project administration; Supervision; Writing - review \& editing.

\section{Geolocation Information:}

The study was conducted at Department of Bioinformatics, Guru Nanak Khalsa College of Arts, Science \& Commerce (Autonomous), Nathalal Parekh Marg, Matunga, Mumbai, Maharashtra, India - 400019.

\section{Data Availability Statement:}

The authors confirm that the data supporting the findings of this study are available within the article.

About the License

(C) The author(s) 2021. The text of this article is open access and licensed under a Creative Commons Attribution 4.0 International License 